\title{
ОБРАТНЫЕ ЗАДАЧИ ТЕПЛОМАССООБМЕНА НА ПРОНИЦАЕМЫХ ПОВЕРХНОСТЯХ ГИПЕРЗВУКОВЫХ ЛЕТАТЕЛЬНЫХ АППАРАТОВ. V. СМЕШАННЫЕ ЗАДАЧИ НА ФРАГМЕНТАХ УЧАСТКА УПРАВЛЕНИЯ
}

\author{
Г. Г. Бильченко, Н. Г. Бильченко \\ Казанский национальный исследовательский технический университет \\ (КНИТУ - КАИ) им. А. Н. Туполева
}

Поступила в редакцию 13.06.2018 г.

\begin{abstract}
Аннотация. Рассматриваются задачи математического моделирования эффективного управления тепломассообменом и трением в ламинарном пограничном слое на проницаемых цилиндрических и сферических поверхностях гиперзвуковых летательных аппаратов. Приводятся постановки смешанных задач на фрагментах участка управления. Обсуждаются вопросы близости в смешанных задачах. Приводится схема алгоритма выбора варианта решаемой задачи.

Ключевые слова: управление, тепломассообмен, трение, ламинарный пограничный слой, проницаемые поверхности, гиперзвуковые течения, прямые задачи, обратные задачи, смешанные задачи.

Annotation. The problems of mathematical modeling of effective control of heat and mass transfer and friction in laminar boundary layer on permeable cylindrical and spherical surfaces of hypersonic aircraft are considered. Mixed problems are formulated for fragments of segment of control. Questions of proximity in mixed problems are discussed. A scheme of algorithm for choosing the variant of the problem being solved is given.

Keywords: control, heat and mass transfer, friction, laminar boundary layer, permeable surfaces, hypersonic flows, direct problems, inverse problems, mixed problems.
\end{abstract}

\section{ВВЕДЕНИЕ}

Являясь продолжением статьи [1], данная работа сохраняет введённые в ней обозначения и использует её библиографический список (начиная с позиции [2]).

Ранее прямые (как обычные, так и экстремальные) [9-12, 14-16] и обратные [2-4] задачи управления ЛПС на проницаемых поверхностях ГЛА рассматривались раздельно и на всём участке управления.

Однако, для обеспечения необходимого сочетания теплозащитных и аэродинамических свойств поверхностей ГЛА, предназначенных для движения в плотных слоях атмосферы, и определяемых назначением конкретного ГЛА,

(c) Бильченко Г. Г., Бильченко Н. Г., 2018 требуется учёт конструкторских и газодинамических ограничений [17], подразумевающих и фрагментацию участка управления, и применение различных управляющих воздействий на его фрагментах.

В данной работе, продолжающей исследования [1-4, 9-12, 14-16]:

1) вводится фрагментация отрезка управления;

2) приводятся постановки смешанных задач;

3) обсуждаются вопросы близости в смешанных задачах;

4) проводится классификация смешанных задач управления ТМО и трением в ЛПС на проницаемых поверхностях ГЛА на фрагментах участка управления;

5) приводится иллюстрирующая классификацию таблица; 


\section{Г. Г. Бильченко, Н. Г. Бильченко}

6) целевая функция $\Psi$ из [1] вводится для кусочно-непрерывных функций $\varphi$;

7) вводится определение парциальных ограничений;

8) приводится схема алгоритма выбора варианта решаемой задачи.

\section{1. ФРАГМЕНТАЦИЯ}

1.1. Пусть $r \geq 1 \oint$ фагментов

$$
X_{\ell}=\left[x_{i_{\hat{\ell}-1}^{\wedge}}^{\wedge} ; x_{i_{\ell}^{\wedge}}^{\wedge}\right] \text { для } \ell=1, \ldots, r
$$

выделены на отрезке $X_{t o t}=[0 ; 1]$ с помощью выбора индексов

$$
i_{0}^{\wedge}=0<i_{1}^{\wedge}<\ldots<i_{r}^{\wedge}=n^{\wedge}
$$

точек сетки управления $X^{\wedge}=\left(x_{j}^{\wedge}\right)_{j=0, \ldots, n^{\wedge}}$. В условиях (9) из [1] индексы

$$
i_{0}^{\vee}=0<i_{1}^{\vee}<\ldots<i_{r}^{\vee}=n^{\vee}
$$

точек сетки наблюдения $X^{\vee}=\left(x_{j}^{\vee}\right)_{j=0, \ldots, n^{\vee}}$ такие, что для всех $\ell=1, \ldots, r$

$$
x_{i_{\ell}^{\vee}}^{\vee}=x_{i_{\ell}^{\wedge}}^{\wedge},
$$

т. е. $X_{\ell}=\left[x_{i_{\ell-1}^{\vee}}^{\vee} ; x_{i_{\ell}^{\vee}}^{\vee}\right]$, определяются однозначно. Обозначим (для $\ell=1, \ldots, r)$

$$
\begin{aligned}
& X_{\ell}^{\wedge}=X^{\wedge} \cap X_{\ell}=\left(x_{j}^{\wedge}\right)_{j=i_{\ell-1}^{\wedge}, \ldots, i_{\ell}^{\wedge}}, \\
& X_{\ell}^{\vee}=X^{\vee} \cap X_{\ell}=\left(x_{j}^{\vee}\right)_{j=i_{\ell-1}^{\vee}, \ldots, i_{\ell}^{\vee}} .
\end{aligned}
$$

Наборы сеток

и интервалов

$$
\begin{aligned}
& X_{(r)}^{\wedge}=\left(X_{\ell}^{\wedge}\right)_{\ell=1, \ldots, r}, \\
& X_{(r)}^{\vee}=\left(X_{\ell}^{\vee}\right)_{\ell=1, \ldots, r}
\end{aligned}
$$

будем называть фрагментациями сеток $X^{\wedge}$, $X^{\vee}$ и интервала $X_{t o t}$, соответственно.

Набор элементов [17] функции на фрагменте $X_{\ell}$ назовём фрагментом функиии.

1.2. Интегральные функционалы $Q, F, N$, вычисляемые на отрезках $X_{\text {tot }}$ и $X_{\ell}$, обозначим $Q_{t o t}, F_{t o t}, N_{t o t}$ и $Q_{\ell}, F_{\ell}, N_{\ell}$, соответственно. $Q_{t o t}, F_{t o t}, N_{t o t}$ будем называть тотальными, а $Q_{\ell}, F_{\ell}, N_{\ell}$ - парииальными. Выполняются соотношения

$$
Q_{t o t}=\sum_{\ell=1}^{r} Q_{\ell}, F_{t o t}=\sum_{\ell=1}^{r} F_{\ell}, N_{t o t}=\sum_{\ell=1}^{r} N_{\ell} .
$$

1.3. Задачи с управлениями на (7) и с вычисляемыми и/или наблюдаемыми параме- трами на (8) назовём задачами на фрагменmax. Поставленная на $X_{t o t}$ задача (ПЗ, ИОЗ, АО3, СО3, ГПЭ3, ОЭ3) введением индексов (2), а за ними (3), преобразуется в набор задач на фрагментах.

Пример 1. Пусть для некоторых $X^{\wedge}$ и $X^{\vee}$ с $n^{\wedge} \geq 2$ задана $\operatorname{ИO}_{m}^{q}\left(\right.$ с $\left.v^{\tau} \geq 1\right)$ :

$$
X_{t o t}:\left(\left(q_{j}^{\vee}\right)_{j=0, \ldots, n^{\vee}}^{I} ;\left(\tau_{j}^{(k)}\right)_{\substack{j=0, \ldots, n^{\wedge} \\ k=0, \ldots, v^{\tau}}}^{I}\right) .
$$

Пусть $r=2$. Выбирая индекс $i_{1}^{\wedge}$ из $\left\{1, \ldots, n^{\wedge}-1\right\}$, определим точку стыка $x_{i_{1}^{\vee}}^{\vee}=x_{i_{1}^{\wedge}}^{\wedge}$ получим фрагментации (7)-(9) и

$$
\begin{gathered}
\left(q_{j}^{\vee}\right)_{j}^{I} \rightarrow\left(q_{j}^{\vee}\right)_{j=0, \ldots, i_{1}^{\vee}}^{A}, \quad\left(q_{j}^{\vee}\right)_{j=i_{1}^{\vee}, \ldots, n^{\vee}}^{B} ; \\
\left(\tau_{j}^{(k)}\right)_{j}^{I} \rightarrow\left(\tau_{j}^{(k)}\right)_{\substack{j=0, \ldots, \hat{i}_{1} \\
k=0, \ldots, v^{\tau}}}^{A},\left(\tau_{j}^{(k)}\right)_{\substack{j=i_{1}^{\wedge}, \ldots, n^{\wedge} \\
k=0, \ldots, v^{\tau}}}^{B} .
\end{gathered}
$$

Пусть на $X^{\wedge}, X^{\vee}$ задана ещё одна ИОЗ $_{m}^{q}$ (с тем же $v^{\tau} \geq 1$ ):

$$
X_{\text {tot }}:\left(\left(q_{j}^{\vee}\right)_{j=0, \ldots, n^{\vee}}^{I I} ;\left(\tau_{j}^{(k)}\right)_{\substack{j=0, \ldots, n^{\wedge} \\ k=0, \ldots, v^{\tau}}}^{I I}\right) .
$$

Она позволяет создать наборы данных:

$$
\begin{aligned}
& \left(q_{j}^{\vee}\right)_{j}^{I I} \rightarrow\left(q_{j}^{\vee}\right)_{j=0, \ldots, i_{1}^{\vee}}^{C}, \quad\left(q_{j}^{\vee}\right)_{j=i_{1}^{\vee}, \ldots, n^{\vee}}^{D} ; \\
& \left(\tau_{j}^{(k)}\right)_{\substack{j \\
k}}^{I I} \rightarrow\left(\tau_{j}^{(k)}\right)_{\substack{j=0, \ldots, \hat{i}_{1}^{\wedge} \\
k=0, \ldots, \nu^{\tau}}}^{C},\left(\tau_{j}^{(k)}\right)_{\substack{j=\hat{i}_{1}^{\wedge}, \ldots, n^{\wedge} \\
k=0, \ldots, \nu^{\tau}}}^{D} .
\end{aligned}
$$

Отметим, что $\quad q_{i_{1}^{\vee}}^{\vee, A}=q_{i_{1}^{\vee}}^{\vee, B}, \quad \tau_{i_{1}^{\wedge}}^{(k), A}=\tau_{i_{1}^{\wedge}}^{(k), B}$, $q_{i_{1}^{\vee}}^{\vee, C}=q_{i_{1}^{\vee}}^{\vee, D}, \tau_{i_{1}^{\wedge}}^{(k), C}=\tau_{i_{1}^{\wedge}}^{(k), D}$.

1.4. Набор задач на фрагментах, рассматриваемый на $X_{t o t}$, будем называть смешанной задачей (далее - СЗ). При этом, набор без ГПЭЗ и ОЭЗ будем называть смешанной неэкстремальной задачей (далее - СНЭЗ), а набор с ГПЭЗ и/или ОЭЗ - смешанной экстремальной задачей (далее - СЭЗ). СЗ допускает две постановки, которые рассматриваются для СНЭЗ в п. 2.3 , п. 2.4 , а для СЭЗ - в п. 3.3, п. 3.4 .

Из полученных в примере 1 фрагментов $\left(q_{j}^{\vee}\right)_{j=0, \ldots, i_{1}^{\vee}}^{A},\left(q_{j}^{\vee}\right)_{j=i_{1}^{\vee}, \ldots, n^{\vee}}^{B},\left(q_{j}^{\vee}\right)_{j=0, \ldots, i_{1}^{\vee}}^{C},\left(q_{j}^{\vee}\right)_{j=i_{1}^{\vee}, \ldots, n^{\vee}}^{D}$ можно сформировать 2 исходных варианта наблюдаемых параметров $q$ : $I=(A, B)$, $I I=(C, D)$, а также 2 новых: $(A, D),(C, B)$. Аналогично для управления $\tau$. Собирая ИОЗ $_{m}^{q}$

$$
X_{1}:\left(\left(q_{j}^{\vee}\right)^{L} ;\left(\tau_{j}^{(k)}\right)^{L}\right) \leftarrow\left(\left(q_{j}^{\vee}\right)^{A} ;\left(\tau_{j}^{(k)}\right)^{A}\right),
$$


Обратные задачи тепломассообмена на проницаемых поверхностях ГЛА. V. ...

$$
X_{2}:\left(\left(q_{j}^{\vee}\right)^{R} ;\left(\tau_{j}^{(k)}\right)^{R}\right) \leftarrow\left(\left(q_{j}^{\vee}\right)^{B} ;\left(\tau_{j}^{(k)}\right)^{B}\right),
$$

получим С3 на $X_{t o t}$, идентичную (11).

1.5. Для каждого $\ell=1, \ldots, r-1$ значения наблюдаемых на $X_{\ell+1}$ параметров $q, f$ в точке стыка $x_{i_{\ell}^{\vee}}^{\vee}$ фрагментов $X_{\ell}=\left[\begin{array}{c}x_{i_{\ell-1}^{\vee}}^{\vee} ; x_{i_{\ell}^{\vee}}^{\vee} \\ v_{\ell}\end{array}\right.$ $X_{\ell+1}=\left[x_{i_{\ell}^{\vee}}^{\vee} ; x_{i_{\ell+1}^{\vee}}^{\vee}\right]$ в С3 должны быть согласованы со значениями таких же параметров, вычисляемых или наблюдаемых на $X_{\ell}$ в этой точке. Так, если в условиях примера 1 взять

$$
\begin{aligned}
& X_{1}:\left(\left(q_{j}^{\vee}\right)^{L} ;\left(\tau_{j}^{(k)}\right)^{L}\right) \leftarrow\left(\left(q_{j}^{\vee}\right)^{A} ;\left(\tau_{j}^{(k)}\right)^{A}\right), \\
& X_{2}:\left(\left(q_{j}^{\vee}\right)^{R} ;\left(\tau_{j}^{(k)}\right)^{R}\right) \leftarrow\left(\left(q_{j}^{\vee}\right)^{D} ;\left(\tau_{j}^{(k)}\right)^{B}\right),
\end{aligned}
$$

то при $q_{i_{1}^{\vee}}^{\vee, A} \neq q_{i_{1}^{\vee}}^{\vee, D}$ полученная С3 окажется переопределённой. Требование вида

$$
y_{i_{\ell}^{\vee}}^{\vee, L}=y_{i_{\ell}^{\vee}}^{\vee, R},
$$

где $y=q$ или $y=f$, является достаточным для корректности постановки.

Для управляющих параметров $m, \tau$ условия согласования заданы в $I^{m}, I^{\tau}, \underline{\Delta m}, \ldots$, $\overline{\Delta \tau}$. Для заданных на $X_{\ell+1}$ параметров $m, \tau$ при $\underline{\Delta m}_{j, k}=\overline{\Delta m}_{j, k}, \underline{\Delta \tau}{ }_{j, k}=\overline{\Delta \tau}_{j, k}$ задача может оказаться переопределённой. Для разыскиваемых на $X_{\ell+1}$ параметров $m^{\sim}, \tau^{\sim}$ при $\underline{\Delta m}_{j, k} \neq \overline{\Delta m}_{j, k}, \quad \underline{\Delta \tau}{ }_{j, k} \neq \overline{\Delta \tau}_{j, k}$ задача будет недоопределённой. Так, если в условиях примера 1 сформировать С3:

$$
\begin{aligned}
& X_{1}:\left(\left(q_{j}^{\vee}\right)^{L} ;\left(\tau_{j}^{(k)}\right)^{L}\right) \leftarrow\left(\left(q_{j}^{\vee}\right)^{A} ;\left(\tau_{j}^{(k)}\right)^{A}\right), \\
& X_{2}:\left(\left(q_{j}^{\vee}\right)^{R} ;\left(\tau_{j}^{(k)}\right)^{R}\right) \leftarrow\left(\left(q_{j}^{\vee}\right)^{B} ;\left(\tau_{j}^{(k)}\right)^{D}\right),
\end{aligned}
$$

то при $\quad \tau_{i_{\hat{\ell}}}^{(k), L}+\overline{\Delta \tau}_{i_{\hat{\ell}}^{\hat{\ell}}, k}=\tau_{i_{\hat{\ell}}^{\hat{\ell}}}^{(k), R}, \quad \underline{\Delta \tau_{i_{\ell}, k}}=\overline{\Delta \tau}_{i_{\hat{\ell}}, k}$, $k=0, \ldots, v^{\tau}, \overline{\Delta \tau}_{i_{\ell}, 0}=0$ получаемая С3 будет корректной.

Выводы. 1) Ограничимся случаем фиксированного размера скачков (п. 3.2 [17]).

2) Для $\ell=1, \ldots, r-1$ наблюдаемые и управляющие параметры на $X_{\ell+1}^{\vee}$ и $X_{\ell+1}^{\wedge}$ следует задавать в точках

$$
\left(x_{j}^{\vee}\right)_{j=i_{\ell}^{\vee}+1, \ldots, i_{\ell_{+1}}^{\vee}} \text { И }\left(x_{j}^{\wedge}\right)_{j=i_{\ell}^{\wedge}+1, \ldots, i_{\ell_{+1}+1}^{\wedge}},
$$

соответственно. В этом случае значения параметров на левой границе фрагмента $X_{\ell+1}$ однозначно определяются значениями на правой границе $X_{\ell}$.

\section{2. СМЕШАННЫЕ}

\section{НЕЭКСТРЕМАЛЬНЫЕ ЗАДАЧИ}

2.1. Пусть заданы: фрагментация (7), управление $s$, числа $p \in[1 ;+\infty]$ и $\varepsilon>0$. Для О3, peализующихся на различных фрагментах, ограничимся случаем единого показателя близости, равного заданному значению $p$. Для ИОЗ и СО3, реализующихся на различных фрагментах, ограничимся случаем единой точности, равной заданному значению $\varepsilon$.

Пусть для каждого $\ell=1, \ldots, r$ два из четырёх параметров $m, \tau, q, f$ заданы (“1”) на фрагменте $X_{\ell}\left(m, \tau\right.$ - на $X_{\ell}^{\wedge}, q, f-$ на $\left.X_{\ell}^{\vee}\right)$, а два на нём свободны (“0”). Оснащая $\delta$ из табл. 1 из [1] индексом $\ell$, получим

$$
\delta_{\ell}^{m}+\delta_{\ell}^{\tau}+\delta_{\ell}^{q}+\delta_{\ell}^{f}=2 .
$$

Пусть для каждого из заданных наблюдаемых параметров указана либо интерполяционная, либо аппроксимационная постановка, т. е. заданы $\delta_{\ell}^{i: q}, \delta_{\ell}^{a: q}, \delta_{\ell}^{i: f}, \delta_{\ell}^{a: f}$, удовлетворяющие (18), (19) из [1]. Найдём

$$
\begin{aligned}
& \delta_{\ell}^{\mathrm{UO}_{m}^{q}}=\delta_{\ell}^{\tau} \cdot \delta_{\ell}^{i: q}, \quad \delta_{\ell}^{\mathrm{AO} 3_{m}^{q}}=\delta_{\ell}^{\tau} \cdot \delta_{\ell}^{a: q}, \\
& \delta_{\ell}^{\mathrm{UO}_{m}^{f}}=\delta_{\ell}^{\tau} \cdot \delta_{\ell}^{i: f}, \delta_{\ell}^{\mathrm{AO}_{m}^{f}}=\delta_{\ell}^{\tau} \cdot \delta_{\ell}^{a: f}, \\
& \delta_{\ell}^{\mathrm{UO} 3_{\tau}^{q}}=\delta_{\ell}^{m} \cdot \delta_{\ell}^{i: q}, \quad \delta_{\ell}^{\mathrm{AO} 3_{\tau}^{q}}=\delta_{\ell}^{m} \cdot \delta_{\ell}^{a: q}, \\
& \delta_{\ell}^{\mathrm{UO}_{\tau}^{f}}=\delta_{\ell}^{m} \cdot \delta_{\ell}^{i: f}, \delta_{\ell}^{\mathrm{AO}_{\tau}^{f}}=\delta_{\ell}^{m} \cdot \delta_{\ell}^{a: f}, \\
& \delta_{\ell}^{\mathrm{nO} 3^{q, f}}=\delta_{\ell}^{i: q} \cdot \delta_{\ell}^{i: f}, \delta_{\ell}^{\mathrm{AO} 3^{q, f}}=\delta_{\ell}^{a: q} \cdot \delta_{\ell}^{a: f}, \\
& \delta_{\ell}^{\mathrm{CO}^{i q, a, f}}=\delta_{\ell}^{i: q} \cdot \delta_{\ell}^{a: f}, \delta_{\ell}^{\mathrm{CO}^{a, q, i f f}}=\delta_{\ell}^{a: q} \cdot \delta_{\ell}^{i: f} \text {, } \\
& \delta_{\ell}^{\Pi 3}=\delta_{\ell}^{m} \cdot \delta_{\ell}^{\tau},
\end{aligned}
$$

а также

$$
\begin{aligned}
& \delta_{\ell}^{\mathrm{O} 3}=\delta_{\ell}^{q} \vee \delta_{\ell}^{f}, \delta_{\ell}^{\mathrm{H} Э 3}=\delta_{\ell}^{\Pi 3}+\delta_{\ell}^{\mathrm{O} 3}, \\
& \delta_{\ell}^{\mathrm{OO} 3}=\left(\delta_{\ell}^{m} \vee \delta_{\ell}^{\tau}\right) \cdot\left(\delta_{\ell}^{q} \vee \delta_{\ell}^{f}\right), \delta_{\ell}^{\text {मО3 }}=\delta_{\ell}^{q} \cdot \delta_{\ell}^{f} \text {, } \\
& \delta_{\ell}^{\mathrm{LO} 3}=\delta_{\ell}^{i} \cdot\left(1-\delta_{\ell}^{a}\right), \delta_{\ell}^{\mathrm{AO} 3}=\left(1-\delta_{\ell}^{i}\right) \cdot \delta_{\ell}^{a},
\end{aligned}
$$

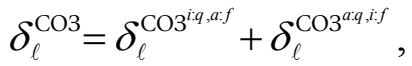

где величины $\delta_{\ell}^{i}, \delta_{\ell}^{a}$ определены в (16), (17) из [1]. Очевидно,

$$
\begin{aligned}
& \delta_{\ell}^{\mathrm{HЭ3}}=1, \delta_{\ell}^{\mathrm{OO} 3}+\delta_{\ell}^{\mathrm{LO} 3}=\delta_{\ell}^{\mathrm{O} 3}, \\
& \delta_{\ell}^{\mathrm{UO} 3_{m}^{q}}+\delta_{\ell}^{\mathrm{hO}_{m}^{f}}+\delta_{\ell}^{\mathrm{hO} 3_{\tau}^{q}}+\delta_{\ell}^{\text {ИO } 3_{\tau}^{f}}+ \\
& +\delta_{\ell}^{\text {ИО } 3^{q, f}}=\delta_{\ell}^{\text {ИО }}, \\
& \delta_{\ell}^{\mathrm{AO}_{m}^{q}}+\delta_{\ell}^{\mathrm{AO}_{m}^{f}}+\delta_{\ell}^{\mathrm{AO}_{\tau}^{q}}+\delta_{\ell}^{\mathrm{AO}_{\tau}^{f}}+ \\
& +\delta_{\ell}^{\mathrm{AOO} 3^{q, f}}=\delta_{\ell}^{\mathrm{AO} 3},
\end{aligned}
$$




$$
\begin{gathered}
\delta_{\ell}^{\mathrm{UO} 3}+\delta_{\ell}^{\mathrm{AO} 3}+\delta_{\ell}^{\mathrm{CO} 3}=\delta_{\ell}^{\mathrm{O} 3}, \\
\delta_{\ell}^{\mathrm{UO} 3_{m}^{q}}+\ldots+\delta_{\ell}^{\mathrm{UO}_{\tau}^{f}}+ \\
+\delta_{\ell}^{\mathrm{AO} 3_{m}^{q}}+\ldots+\delta_{\ell}^{\mathrm{AO}_{\tau}^{f}}=\delta_{\ell}^{\mathrm{OO} 3}, \\
\delta_{\ell}^{\mathrm{UO}^{q, f}}+\delta_{\ell}^{\mathrm{AO} 3^{q, f}}+\delta_{\ell}^{\mathrm{CO} 3}=\delta_{\ell}^{\text {ДO3 }} .
\end{gathered}
$$

В условиях (13) на каждом фрагменте $X_{\ell}$ peaлизуется одна из 12 О3, перечисленных в табл. 2 и 3 из [1], или П3: в (14) в точности одна $\delta_{\ell}$ обращается в "1". Суммируя $\delta_{\ell}$ в $(14)$ и в (15) по $\ell$ от 1 до $r$, найдём количества видов задач на фрагментах:

$$
\begin{aligned}
v^{\mathrm{ИO} 3_{m}^{q}} & =\sum_{\ell=1}^{r} \delta_{\ell}^{\mathrm{LO} 3_{m}^{q}}, \ldots, v^{\Pi 3}=\sum_{\ell=1}^{r} \delta_{\ell}^{\Pi 3}, \\
v^{\mathrm{O} 3} & =\sum_{\ell=1}^{r} \delta_{\ell}^{\mathrm{O} 3}, \ldots, v^{\mathrm{CO} 3}=\sum_{\ell=1}^{r} \delta_{\ell}^{\mathrm{CO} 3} .
\end{aligned}
$$

2.2. Постановки ОЗ на $X_{\ell}$ предполагают введение расстояний. Пусть при $p=+\infty$ :

$$
R_{\infty, \ell}\left(y^{\sim} ; y^{\vee}\right)=\max _{j=i_{\ell-1}^{\vee}, \ldots, i_{\ell}^{\vee}}\left|\tilde{y_{j}^{\sim}}-y_{j}^{\vee}\right|,
$$

при $p \in[1 ;+\infty)$ :

$$
R_{p, \ell}\left(y^{\sim} ; y^{\vee}\right)=\left(\sum_{j=i_{\ell-1}^{\vee}}^{i_{\ell}^{\vee}}\left|y_{j}^{\sim}-y_{j}^{\vee}\right|^{p}\right)^{1 / p},
$$

где $y=q$ или $y=f$. Для $\sigma \in\{a ; i\}$ введём при $p=+\infty$ :

$$
\begin{gathered}
D_{\infty, \ell}^{\sigma}=\max \left\{\delta_{\ell}^{\sigma: q} \cdot R_{\infty, \ell}\left(q^{\sim} ; q^{\vee}\right),\right. \\
\left.\delta_{\ell}^{\sigma: f} \cdot R_{\infty, \ell}\left(f^{\sim} ; f^{\vee}\right)\right\}, \\
R_{\infty}^{\sigma}\left(\left(q^{\sim} ; f^{\sim}\right) ;\left(q^{\vee} ; f^{\vee}\right)\right)=\max _{\ell=1, \ldots, r} D_{\infty, \ell}^{\sigma},
\end{gathered}
$$

при $p \in[1 ;+\infty)$ :

$$
\begin{gathered}
D_{p, \ell}^{\sigma}=\left(\delta_{\ell}^{\sigma: q} \cdot R_{p, \ell}^{p}\left(q^{\sim} ; q^{\vee}\right)+\right. \\
\left.+\delta_{\ell}^{\sigma: f} \cdot R_{p, \ell}^{p}\left(f^{\sim} ; f^{\vee}\right)\right)^{1 / p}, \\
E_{p, \ell}^{\sigma}=\left(\delta_{\ell}^{\sigma: q} \cdot \delta_{\ell+1}^{\sigma: q}\left|q_{i_{\ell}^{\sim}}^{\sim}-q_{i_{\ell}^{\vee}}^{\vee}\right|^{p}+\right. \\
\left.+\delta_{\ell}^{\sigma: f} \cdot \delta_{\ell+1}^{\sigma: f}\left|f_{i_{\ell}^{\vee}}^{\sim}-f_{i_{\ell}^{\vee}}^{\vee}\right|^{p}\right)^{1 / p}, \\
R_{p}^{\sigma}\left(\left(q^{\sim} ; f^{\sim}\right) ;\left(q^{\vee} ; f^{\vee}\right)\right)= \\
=\left[\sum_{\ell=1}^{r}\left(D_{p, \ell}^{\sigma}\right)^{p}-\sum_{\ell=1}^{r-1}\left(E_{p, \ell}^{\sigma}\right)^{p}\right]^{1 / p},
\end{gathered}
$$

где $\delta_{\ell}^{i: q}, \delta_{\ell}^{a: q}, \delta_{\ell}^{i: f}, \delta_{\ell}^{a: f}$ из табл. 2, 3 из [1].
Формулы (19) и (21) позволяют формально свести постановки различных О3 на $X_{\ell}$ (в том числе и ПЗ, для которой $\delta_{\ell}^{i: q}=\delta_{\ell}^{a: q}=\delta_{\ell}^{i: f}=$ $\left.=\delta_{\ell}^{a: f}=0\right) \kappa \mathrm{O} 3_{(m, \tau)}^{(q, f)}$.

Формулы (20) и (23) позволяют формально свести постановки различных О3 на $X_{(r)}$ (в том числе и ПЗ) к $3_{(m, \tau)}^{(q, f)}$ на $X_{t o t}$.

2.3. Простая СЗ предполагает последовательное от $\ell=1$ до $\ell=r$ (т. е. вниз по потоку) решение задач на фрагментах. Другими словами,

если на $X_{\ell}$ задана ПЗ $\left(\delta_{\ell}^{m}=\delta_{\ell}^{\tau}=1\right)$, то на нём вычисляются $q$ и $f$;

если на $X_{\ell}$ задана ИОЗ $\left(\delta_{\ell}^{i}=1, \delta_{\ell}^{a}=0\right)$, то свободные на нём параметры $m^{\sim}$ или/и $\tau^{\sim}$ разыскиваются в соответствии с условиями (10) или/и (11) из [1], а также

$$
D_{p, \ell}^{i} \leq \varepsilon
$$

если на $X_{\ell}$ задана AO3 $\left(\delta_{\ell}^{i}=0, \delta_{\ell}^{a}=1\right)$, то свободные на нём параметры $m^{\sim}$ или/и $\tau^{\sim}$ разыскиваются, как приближённое решение экстремальной задачи

$$
\begin{aligned}
& \inf _{m^{\sim}} D_{p, \ell}^{a} \text { при } \delta_{\ell}^{m}=0, \delta_{\ell}^{\tau}=1, \\
& \inf _{\tau^{\sim}} D_{p, \ell}^{a} \text { при } \delta_{\ell}^{m}=1, \delta_{\ell}^{\tau}=0, \\
& \inf _{m^{\sim}, \tau^{\sim}} D_{p, \ell}^{a} \quad \text { при } \delta_{\ell}^{m}=0, \delta_{\ell}^{\tau}=0
\end{aligned}
$$

при выполнении условий (10), (11) из [1];

если на $X_{\ell}$ задана СО3 $\left(\delta_{\ell}^{a}=\delta_{\ell}^{i}=1\right)$, то параметры $m^{\sim}, \tau^{\sim}$ разыскиваются на нём, как приближённое решение экстремальной задачи (27) при выполнении условий (10), (11) из [1] и (24).

2.4. Сложная СЗ предполагает одновременное отыскание управлений $m^{\sim}$ и $\tau^{\sim}$, удовлетворяющих условиям (10) и (11) из [1], на всех тех фрагментах $X_{\ell}^{\wedge}$, где они не заданы $\left(\delta_{\ell}^{m}=0\right.$ и/или $\left.\delta_{\ell}^{\tau}=0\right)$, как приближённых решений экстремальной задачи

$$
\inf _{m^{\sim}, \tau^{\sim}} R_{p}^{a}\left(\left(q^{\sim} ; f^{\sim}\right) ;\left(q^{\vee} ; f^{\vee}\right)\right)
$$

при условии

$$
R_{p}^{i}\left(\left(q^{\sim} ; f^{\sim}\right) ;\left(q^{\vee} ; f^{\vee}\right)\right) \leq \varepsilon .
$$

2.5. В табл. 1 в строках «пз», «иоз», «аоз», «соз» и в столбцах «пз», «иоз», «аоз», «соз» приведены результаты сочетаний пар задач на фрагментах. Здесь «иоз» и «ИОЗ» (или 
«аоз» и « $\mathrm{AO} 3 »)$ - различающиеся (параметрами) иоз (аоз); «соз» и «СОЗ»- различающиеся постановками соз. В частности, «исз» («асз») - С3, содержащие обратные задачи только в интерполяционной (в аппроксимационной) постановке и, возможно, ПЗ. В остальной части (три нижних строки и три правых столбца) приведены результаты сочетаний с «исз», «асз», «сз».

Таблица 1

\begin{tabular}{|c|c|c|c|c|c|c|c|}
\hline & П3 & ИОз & ao3 & $\mathrm{CO} 3$ & ИСз & ac3 & c3 \\
\hline Пз & п3 & исз & ac3 & C3 & ИС 3 & ac3 & C3 \\
\hline ИОЗ & \multirow{2}{*}{ ИСЗ } & иоз & \multirow{2}{*}{$\mathrm{c} 3$} & \multirow{2}{*}{ c3 } & \multirow{2}{*}{ ИСз } & \multirow{2}{*}{ c3 } & \multirow{2}{*}{ C3 } \\
\hline ИОЗ & & ИСЗ & & & & & \\
\hline ao3 & \multirow{2}{*}{ ac3 } & \multirow{2}{*}{ c3 } & ao3 & \multirow{2}{*}{ c3 } & \multirow{2}{*}{ C3 } & \multirow{2}{*}{ ac3 } & \multirow[b]{2}{*}{ C3 } \\
\hline $\mathrm{AO} 3$ & & & ac3 & & & & \\
\hline $\mathrm{CO} 3$ & \multirow[b]{2}{*}{ c3 } & \multirow{2}{*}{ C3 } & \multirow{2}{*}{$\mathrm{c} 3$} & $\mathrm{CO} 3$ & \multirow{2}{*}{ C3 } & \multirow{2}{*}{$\mathrm{C} 3$} & \multirow[b]{2}{*}{ C3 } \\
\hline $\mathrm{CO} 3$ & & & & C3 & & & \\
\hline ИСз & ИСЗ & ИСЗ & C3 & c3 & ИС 3 & c3 & C3 \\
\hline ac3 & ac3 & c3 & ac3 & C3 & C3 & ac3 & C3 \\
\hline C3 & C3 & C3 & C3 & C3 & C3 & c3 & C3 \\
\hline
\end{tabular}

2.6. Замечания. 1) Если на всех фрагментах заданы ПЗ, т. е. $v^{\Pi 3}=r$, то СЗ является обычной ПЗ, фрагментация не влияет на получение $q, f$.

2) Если на всех фрагментах заданы ИОЗ (и, возможно, ПЗ), т. е.

$$
v^{\text {иОз }} \geq 1, v^{\mathrm{AO} 3}=0, v^{\mathrm{CO} 3}=0,
$$

то СЗ является ИСЗ. Её простая постановка использует $v^{\text {ИОЗ } у с л о в и и ̆ ~(24), ~ а ~ с л о ж н а я ~-~ у с-~}$ ловие (29).

При $p=+\infty$ решения ИСЗ в этих постановках не отличаются.

При $p \in[1 ;+\infty)$ определим

$$
\varepsilon_{v}=\left(v^{\text {иОЗ }}\right)^{1 / p} \cdot \varepsilon .
$$

Если решение (простой) ИСЗ с $\varepsilon$ в условиях (24) существует, то оно будет решением (сложной) ИСЗ с $\varepsilon_{v}$ в (29). Если решение (сложной) ИСЗ с $\varepsilon$ в (29) существует, то оно будет и решением (простой) ИСЗ с $\varepsilon$ в условиях (24).

3) Для $r>1$ при наличии АОЗ или СО3, т. е.

$$
v^{\mathrm{AO} 3} \geq 1 \text { или } v^{\mathrm{CO} 3} \geq 1,
$$

решения простой С3 и сложной С3 могут различаться. Решение простой С3, может быть использовано в качестве начального приближения для решения сложной С3.

4) Формулы (19)-(23), в частности, поправка (22), построены с учётом (12).

\section{3. СМЕШАННЫЕ ЭКСТРЕМАЛЬНЫЕ ЗАДАЧИ}

3.1. Пусть заданы: фрагментация (7), управление $s$, числа $p \in[1 ;+\infty]$ и $\varepsilon>0$.

Пусть для каждого $\ell=1, \ldots, r$ один или два из четырёх параметров $m, \tau, q, f$ заданы на фрагменте $X_{\ell}$, а остальные на нём свободны, т. е. в отличие от п. 2.1

$$
\delta_{\ell}^{m}+\delta_{\ell}^{\tau}+\delta_{\ell}^{q}+\delta_{\ell}^{f} \in\{1 ; 2\} .
$$

Будем предполагать, что хотя бы на одном фрагменте присутствует экстремальная задача (далее - ЭЗ):

$$
\delta_{\ell}^{m}+\delta_{\ell}^{\tau}+\delta_{\ell}^{q}+\delta_{\ell}^{f}=1 .
$$

Пусть для каждого из заданных наблюдаемых параметров указана либо интерполяционная (в частности, для ОЭЗ), либо аппроксимационная постановка, т. е. заданы $\delta_{\ell}^{i: q}, \delta_{\ell}^{a: q}$, $\delta_{\ell}^{i: f}, \delta_{\ell}^{a: f}$, удовлетворяющие (18), (19) из [1]. Найдём $\delta_{\ell}$ из (14), (15) и

$$
\begin{aligned}
& \delta_{\ell}^{\Gamma \Pi \ni 3_{m}^{O F}}=\left(1-\delta_{\ell}^{m}\right) \cdot \delta_{\ell}^{\tau} \cdot\left(1-\delta_{\ell}^{q}\right) \cdot\left(1-\delta_{\ell}^{f}\right),
\end{aligned}
$$

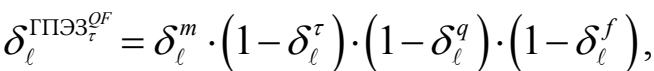

$$
\begin{aligned}
& \delta_{\ell}^{\mathrm{O}^{q 3^{q F}}}=\left(1-\delta_{\ell}^{m}\right) \cdot\left(1-\delta_{\ell}^{\tau}\right) \cdot \delta_{\ell}^{i \cdot q} \cdot\left(1-\delta_{\ell}^{f}\right) \text {, } \\
& \delta_{\ell}^{\mathrm{O} 3^{O f}}=\left(1-\delta_{\ell}^{m}\right) \cdot\left(1-\delta_{\ell}^{\tau}\right) \cdot\left(1-\delta_{\ell}^{q}\right) \cdot \delta_{\ell}^{i: f} \text {, } \\
& \delta_{\ell}^{\Gamma \Pi \ni 3^{Q F}}=\delta_{\ell}^{\Gamma \Pi \ni 3_{m}^{O F}}+\delta_{\ell}^{\Gamma \Pi \ni 3_{\tau}^{Q F}} \text {, } \\
& \delta_{\ell}^{\mathrm{O} 3^{Q F}}=\delta_{\ell}^{\mathrm{O} 3^{q F}}+\delta_{\ell}^{\mathrm{O} \ni 3^{Q f}} \text {, } \\
& \delta_{\ell}^{\ni 3^{O F}}=\delta_{\ell}^{\Gamma \Pi \ni 3^{Q F}}+\delta_{\ell}^{\mathrm{O} \ni 3^{Q F}} .
\end{aligned}
$$

В условиях $(30) \delta_{\ell}^{\text {нэ3 }}+\delta_{\ell}^{\text {э3 }^{O F}}=1$, и на каждом фрагменте может быть задана или одна из 13 НЭ3, перечисленных в п. 2.1, или одна из двух ГПЭЗ, обобщающих ПЭЗ из табл. 4 [1], или одна из двух ОЭЗ из табл. 6 [1]. Таким образом, в (14), (32) в точности одна $\delta_{\ell}$ обращается в "1". Суммируя $\delta_{\ell}$ в $(32)$ и в $(33)$ по $\ell$ от 1 до $r$, найдём количества видов ЭЗ на фрагментах:

$$
\begin{aligned}
& v^{\Gamma \Pi \ni 3_{m}^{O F}}=\sum_{\ell=1}^{r} \delta_{\ell}^{\Gamma \Pi \ni 3_{m}^{O F}}, v^{\Gamma \Pi \ni 3_{\tau}^{O F}}=\sum_{\ell=1}^{r} \delta_{\ell}^{\Gamma \Pi \ni 3_{\tau}^{O F}}, \\
& v^{\mathrm{O}^{93^{q F}}}=\sum_{\ell=1}^{r} \delta_{\ell}^{\mathrm{O} \ni 3^{q F}}, v^{\mathrm{O} 3^{O f}}=\sum_{\ell=1}^{r} \delta_{\ell}^{\mathrm{O} 3^{O f}},
\end{aligned}
$$




\section{Г. Г. Бильченко, Н. Г. Бильченко}

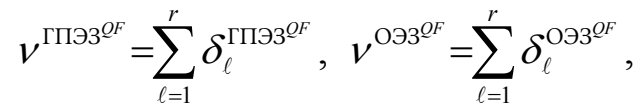

$$
\begin{aligned}
& v^{\ni 3^{Q F}}=\sum_{\ell=1}^{r} \delta_{\ell}^{\ni 3^{Q F}} .
\end{aligned}
$$

3.2. Если задана фрагментация (7), то

$$
\Psi_{t o t}=\sum_{\ell=1}^{r} \Psi_{\ell},
$$

где

$$
\Psi_{\ell}=\sum_{j=i_{\ell-1}^{\wedge}+1}^{i_{\hat{\ell}}} \Psi\left(\left[x_{j-1}^{\wedge} ; x_{j}^{\wedge}\right] ; m, \tau, s ; \varphi\right) .
$$

В СЭЗ для учёта только фрагментов с ЭЗ введём уточнённую целевую функцию

$$
\Psi_{\text {tot }}^{*}=\sum_{\ell=1}^{r} \delta_{\ell}^{\ni 3^{Q F}} \cdot \Psi_{\ell} .
$$

Формулы (42) [1], (35), (37) позволяют формально свести ГПЭЗ к ОЭЗ (для ГПЭЗ $\delta_{\ell}^{i}=0$, нет ограничений на число решений (48), (49) [1], а формально свободный второй параметр $m$ или $\tau$ следует зафиксировать с помощью (24)-(26) из [1]).

Таким образом, далее будем предполагать, что в дополнение к перечисленным в п. 3.1 параметрам на $X^{\wedge}$ с помощью элементов задана кусочно-непрерывная ограниченная функция $\varphi(x)$, у которой для всех $\ell=1, \ldots, r$ при $\delta_{\ell}^{\text {Оэз }{ }^{q F}}=1$ число решений (48) [1] на $X_{\ell}-$ конечно, а при $\delta_{\ell}^{\text {оэз }}=1$ число решений (49) [1] $X_{\ell}$ - конечно.

3.3. Постановка (базовой) простой СЭ3 $3^{(Q, F)}$ с кусочно-непрерывной $\varphi$ предполагает последовательное от $\ell=1$ до $\ell=r$ решение задач на фрагментах. Другими словами,

если на $X_{\ell}$ задана ПЗ или О3 $\left(\delta_{\ell}^{\text {нэз }}=1\right)$, то следует действовать аналогично п. 2.3;

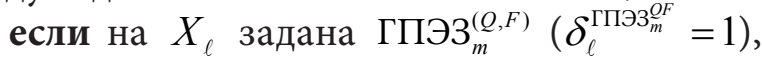
то требуется в условиях (10) из [1] найти управление $m^{\sim}$ и значение

$$
\Psi_{\ell}^{\sim}=\Psi\left(X_{\ell} ; m^{\sim}, \tau, s ; \varphi\right),
$$

как приближённое решение задачи

$$
\inf _{m^{\sim}} \Psi\left(X_{\ell} ; m^{\sim}, \tau, s ; \varphi\right)
$$

минимизации на $X_{\ell}$ функционала (36);

если на $X_{\ell}$ задана ГПЭ3 ${ }_{\tau}^{(Q, F)}\left(\delta_{\ell}^{\Gamma П Э 3_{\tau}^{O F}}=1\right)$, то требуется в условиях (11) из [1] найти управление $\tau^{\sim}$ и значение

$$
\Psi_{\ell}^{\sim}=\Psi\left(X_{\ell} ; m, \tau^{\sim}, s ; \varphi\right),
$$

как приближённое решение задачи

$$
\inf _{\tau^{\sim}} \Psi\left(X_{\ell} ; m, \tau^{\sim}, s ; \varphi\right)
$$

минимизации на $X$, функционала (36);

если на $X_{\ell}$ задана $\mathrm{OЭ3}_{(m, \tau)}^{(q, F)}$ или $\mathrm{OЭ3}_{(m, \tau)}^{(Q, f)}$ $\left(\delta_{\ell}^{\mathrm{оэ}^{9 F}}=1\right.$ или $\left.\delta_{\ell}^{\mathrm{Oэ}^{\varrho f}}=1\right)$, то требуется в условиях (10) и (11) [1] найти управления $m^{\sim}, \tau^{\sim}$ и значение

$$
\Psi_{\ell}^{\sim}=\Psi\left(X_{\ell} ; m^{\sim}, \tau^{\sim}, s ; \varphi\right),
$$

как приближённое решение задачи

$$
\inf _{m^{\sim}, \tau^{\sim}} \Psi\left(X_{\ell} ; m^{\sim}, \tau^{\sim}, s ; \varphi\right)
$$

минимизации на $X_{\ell}$ функционала (36) при выполнении (24).

3.4. В СЭ3 ${ }^{(Q, F)}$ в наборе задач на фрагментах обязаны быть ГПЭЗ или ОЭЗ, следовательно, постановка сложной СЭЗ должна включать оптимизацию (37) на $X_{t o t}$. Согласно п. 2.4, если в наборе задач на фрагментах присутствует AO3 или СО3, то постановка сложной С3 должна включать оптимизацию (28) на $X_{t o t}$. Но функция (28) является неотрицательной в отличие от функции (37). Поэтому результатом смешивания задач с (28) и (37) будет задача двухкритериальной оптимизации. Ограничимся рассмотрением однокритериальных задач без АОЗ или СО3.

Постановка (базовой) сложной (однокритериальной) $C Э 3^{(Q, F)}$ с кусочно-непрерывной $\varphi$ предполагает одновременное отыскание управлений $m^{\sim}$ и $\tau^{\sim}$, удовлетворяющих условиям (10) и (11) из [1], на всех тех фрагментах $X_{\ell}^{\wedge}$, где они не заданы $\left(\delta_{\ell}^{m}=0\right.$ и/или $\left.\delta_{\ell}^{\tau}=0\right)$, как приближённых решений экстремальной задачи

$$
\inf _{m^{\sim}, \tau^{\sim}} \Psi_{t o t}^{*}
$$

при условии (29).

3.5. Замечания. 1) Решение простой СЭЗ может быть использовано в качестве начального приближения для решения сложной СЭЗ.

2) В [13] были поставлены три СЭЗ, сочетающие ${ }^{q}{ }_{m}^{q}$ и $П Э 3_{m}^{Q}$ для случая $X^{\wedge}=X^{\vee}$. 
Обратные задачи тепломассообмена на проницаемых поверхностях ГЛА. V. ...

\section{4. ПАРЦИАЛЬНЫЕ ОГРАНИЧЕНИЯ}

Аналогично определённым на $X_{t o t}$ moтальным ограничениям (33)-(35) из [1], фрагментация позволяет ввести на $X_{\ell}$ парииальные ограничения для $Q_{\ell}, F_{\ell}, N_{\ell}$.

4.1. Базовые постановки ГПЭЗ, ОЭЗ, а также AO3, СО3, рассмотренные в [1] на $X_{t o t}$, могут быть дополнены ограничениями на фрагментах.

Пример 2. Вариант ПЭЗ ${ }_{m}^{Q}(\underline{31})$ из [1]: для заданных $I^{m}, \tau, s, I^{N_{t o t}}=\left[\underline{N}_{t o t} ; \bar{N}_{t o t}\right]$ при введении фрагментации (7) и задании

$$
I^{N_{1}}=\left[\underline{N}_{1} ; \bar{N}_{1}\right], \ldots, I^{N_{r}}=\left[\underline{N}_{r} ; \bar{N}_{r}\right]
$$

требуется в условиях (10), (33) [1] и

$$
\begin{aligned}
& N_{1} \in I^{N_{1}}, \\
& N_{r} \in I^{N_{r}},
\end{aligned}
$$

где $N_{\ell}=N\left(X_{\ell} ; m^{\sim}, \tau, s\right)$, найти $m^{\sim}$, как приближённое решение задачи (31) [1] на $X_{\text {tot }}$. Для ПЭ3 ${ }_{m}^{F}$ - аналогично. Для ПЭЗ $\tau_{\tau}^{Q}$ и $П Э 3_{\tau}^{F}$ используется $N_{\ell}=N\left(X_{\ell} ; m, \tau^{\sim}, s\right)$.

Некорректный выбор $I^{N_{\text {tot }}}, I^{N_{1}}, \ldots, I^{N_{r}}$ приводит к опустошению области допустимых решений. В частности, с учётом (10), должны выполняться условия

$$
\begin{aligned}
& \underline{N}_{t o t} \leq \sum_{\ell=1}^{r} \bar{N}_{\ell}, \\
& \sum_{\ell=1}^{r} \underline{N}_{\ell} \leq \bar{N}_{t o t} .
\end{aligned}
$$

Для $Q$ и $F$ - аналогично. Отметим, что условия вида $\left(45_{1}\right), \ldots,\left(45_{r}\right)$ проще, чем (38) из [1].

4.2. Невырожденные (т. е. $\underline{N}_{\ell}<\bar{N}_{\ell}$ для $\ell=1, \ldots, r)$ условия $\left(45_{1}\right), \ldots,\left(45_{r}\right)$ определяют в пространстве $r$ переменных $\left(N_{1}, \ldots, N_{r}\right)$ внутренность $2 r$-гранника

$$
I^{N_{1}} \times \cdots \times I^{N_{r}},
$$

включая его границу. Невырожденные (т. е. $\underline{N}_{t o t}<\bar{N}_{t o t}$ ) условия (33) [1] при

$$
\sum_{\ell=1}^{r} \underline{N}_{\ell}<\underline{N}_{t o t}
$$

и

$$
\bar{N}_{t o t}<\sum_{\ell=1}^{r} \bar{N}_{\ell}
$$

определяют две параллельные гиперплоскости, отсекающие вершины с координатами $\left(\underline{N}_{1}, \ldots, \underline{N}_{r}\right)$ и $\left(\bar{N}_{1}, \ldots, \bar{N}_{r}\right)$, т. е. добавляются ещё две $(r-1)$-мерные грани.

Если для некоторого $j \in\{1, \ldots, r\}$

$$
\underline{N}_{j}<\left(\underline{N}_{t o t}-\sum_{\ell=1}^{r} \bar{N}_{\ell}\right)+\bar{N}_{j},
$$

то часть $\underline{N}_{j} \leq N_{j}$ условия (45 $)$ удовлетворяется, а соответствующая уравнению $N_{j}=\underline{N}_{j}$ гипергрань отсекается.

Если для некоторого $j \in\{1, \ldots, r\}$

$$
\left(\bar{N}_{t o t}-\sum_{\ell=1}^{r} \underline{N}_{\ell}\right)+\underline{N}_{j}<\bar{N}_{j},
$$

то часть $N_{j} \leq \bar{N}_{j}$ условия $\left(45_{j}\right)$ удовлетворяется, а соответствующая уравнению $N_{j}=N_{j}$ гипергрань отсекается.

Пример 3. На рис. 1 для $r=2$ изображены линии, соответствующие тотальным ограничениям

$$
N_{\text {tot }} \in I^{N_{\text {tot }}} \text { для } I^{N_{\text {tot }}}=[0,6 ; 1,6]
$$

и парциальным ограничениям

$$
\begin{aligned}
& N_{1} \in I^{N_{1}} \text { для } I^{N_{1}}=[0,225 ; 1,225], \\
& N_{2} \in I^{N_{2}} \text { для } I^{N_{2}}=[0,175 ; 0,575] .
\end{aligned}
$$

Линии 1-10 соответствуют уравнениям

$$
\begin{aligned}
& \text { 1: } N_{1}+N_{2}=\underline{N}_{t o t} ; \quad 2: N_{1}+N_{2}=\bar{N}_{t o t} \text {; } \\
& \text { 3: } N_{1}=\underline{N}_{1} ; \quad 4: N_{1}=\bar{N}_{1} \text {; } \\
& \text { 5: } N_{2}=\underline{N}_{2} ; \quad 6: N_{2}=\bar{N}_{2} \text {; } \\
& \text { 7: } N_{1}+N_{2}=\underline{N}_{1}+\underline{N}_{2} \text {; } \\
& \text { 8: } N_{1}+N_{2}=\bar{N}_{1}+\bar{N}_{2} \text {; } \\
& \text { 9: } N_{1}+N_{2}=\underline{N}_{1}+\bar{N}_{2} \text {; } \\
& \text { 10: } N_{1}+N_{2}=\bar{N}_{1}+\underline{N}_{2} \text {; }
\end{aligned}
$$

стрелки при 1-8 указывают положение полуплоскостей - решений соответствующих уравнениям неравенств. Взаимное расположение линий 9 и 10 зависит от знака $\left(\bar{N}_{1}+\underline{N}_{2}\right)-\left(\underline{N}_{1}+\bar{N}_{2}\right)$. Взаимное расположение линий 1 и 8 соответствует неравенству (46), линий 7 и 2 - неравенству (47). Размещение линии 1 между линиями 7 и 9 и линии 2 между линиями 8 и 10 соответствуют случаю всех активных ограничений: как $\left(45_{1}\right),\left(45_{2}\right)$, так и (33) [1]. 


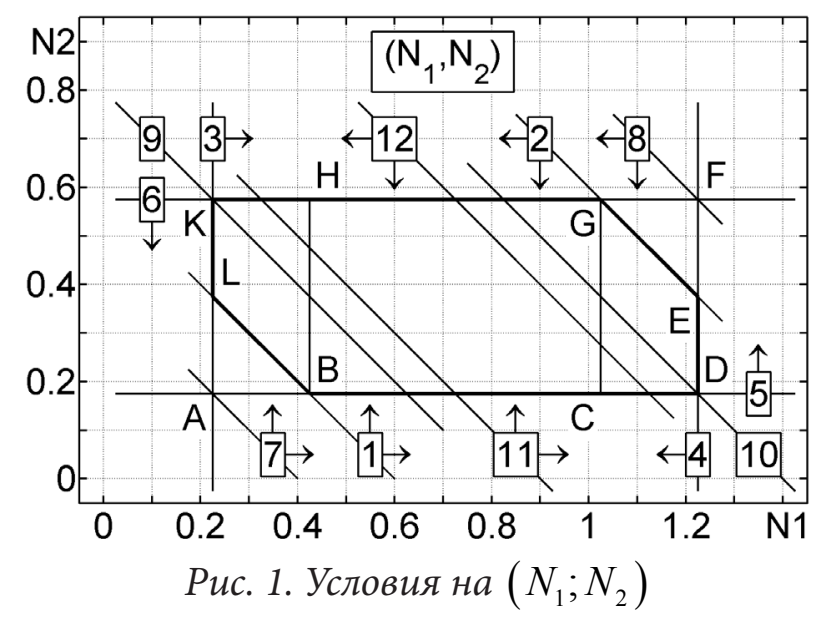

Геометрическое место точек $\left(N_{1} ; N_{2}\right)$, удовлетворяющих всем ограничениям, представляет собой выпуклую замкнутую область внутренность многоугольника $B D E G K L$, включая его границу.

Линии 11, 12 соответствуют уравнениям

11: $N_{1}+N_{2}={\underline{N^{\prime}}}_{t o t} ;$ 12: $N_{1}+N_{2}={\overline{N^{\prime}}}_{t o t}$, где $\underline{N}_{\text {tot }}^{\prime}=0,9$ и ${\overline{N^{\prime}}}_{\text {tot }}=1,3$; стрелки при 11 и 12 определяют положение полосы $N_{t o t} \in I^{N_{t o t}^{\prime}}$. При $j=1$ (для $I^{N_{t o t}^{\prime}}$ ) выполняются (50) и (51), линии 11 и 12 отсекают грани $A K$ и $D F$.

4.3. При использовании условий (33) [1] в С3 в простой постановке если

$$
\underline{N}_{t o t} \leq \sum_{\ell=1}^{r} \underline{N}_{\ell} \quad \text { и } \quad \sum_{\ell=1}^{r} \bar{N}_{\ell} \leq \bar{N}_{t o t},
$$

то тотальные условия выполняются автоматически, и на каждом фрагменте $X_{\ell}$ для $\ell$ от 1 до $r$ используется только одно парциальное условие: $\left(45_{\ell}\right)$.

Если вместо (52) выполняется (48) или/и (49), то на каждом фрагменте $X_{\ell}$ для $\ell$ от 1 до $r$ требуется определить границы допустимых $N_{\ell}$, т. е. решить задачи

$$
\underline{N}_{\ell}^{*}=\min _{N_{\ell}, \ldots, N_{r}} N_{\ell} \quad \text { или } / \text { и } \quad \bar{N}_{\ell}^{*}=\max _{N_{\ell}, \ldots, N_{r}} N_{\ell}
$$

с ограничениями $\left(45_{\ell}\right), \ldots,\left(45_{r}\right)$ и

$$
\begin{aligned}
& \underline{N}_{t o t} \leq S_{\ell, N^{\sim}}^{-}+\sum_{j=\ell}^{r} N_{j}, \\
& S_{\ell, N^{\sim}}^{-}+\sum_{j=\ell}^{r} N_{j} \leq \bar{N}_{t o t},
\end{aligned}
$$

где $S_{\ell, N^{\sim}}^{-}=\sum_{j=1}^{\ell-1} N_{j}^{\sim}$, а $N_{j}^{\sim}$ для $j$ от 1 до $\ell-1$ найдены в процессе решения ПЗ, ОЗ, ГПЭЗ или ОЭЗ на $j$-м фрагменте. Тогда

$$
\begin{aligned}
& \underline{N}_{\ell}^{*}=\max \left\{\underline{N}_{\ell} ; \underline{N}_{t o t}-S_{\ell, N^{\sim}}^{-}-S_{\ell, \bar{N}}^{+}\right\}, \\
& \bar{N}_{\ell}^{*}=\min \left\{\bar{N}_{\ell} ; \bar{N}_{t o t}-S_{\ell, N^{-}}^{-}-S_{\ell, \underline{N}}^{+}\right\},
\end{aligned}
$$

где $S_{\ell, \underline{N}}^{+}=\sum_{j=\ell+1}^{r} \underline{N}_{j}, S_{\ell, \bar{N}}^{+}=\sum_{j=\ell+1}^{r} \bar{N}_{j}$.

В условиях примера 3 если значение $N_{1}^{\sim} \in[0,425 ; 1,025]$, то точка $\left(N_{1}^{\sim} ; N_{2}\right)$ попадает в область $B C G H$, а $N_{2} \in I^{N_{2}}$; если $N_{1}^{\sim} \in[0,225 ; 0,425]$, то точка попадает в область $B H K L$, a $N_{2} \in\left[\underline{N}_{t o t}-N_{1}^{\sim} ; \bar{N}_{2}\right] ;$ если $N_{1}^{\sim} \in[1,025 ; 1,225]$, то точка попадает в область $C D E G$, а $N_{2} \in\left[\underline{N}_{2} ; \bar{N}_{t o t}-N_{1}^{\sim}\right]$.

\section{5. СХЕМА АЛГОРИТМА ВЫБОРА ВАРИАНТА РЕШАЕМОЙ ЗАДАЧИ}

\section{1. Приведём схему алгоритма.}

1. Чтение неизменяемых параметров: тип тела (цилиндр, сфера), радиус тела $R$ [м], высота полёта $H$ [км], скорость полёта $M_{\infty}$. Вычисление по $H$ параметров стандартной атмосферы. Выбор по $M_{\infty}$ [9] расчётной модели газа (совершенный, диссоциирующий, электропроводящий).

2. Чтение сетки $X^{\times}$интегрирования [3]. Чтение сеток $X^{\wedge}, X^{\vee}, X_{(r)}^{\wedge}$. Диагностика $X_{(r)}^{\wedge}$ и $X^{\wedge} \subseteq X^{\vee} \subseteq X^{\times}$.

3. Чтение для $X_{t o t}$ и для каждого фрагмента ограничений $I^{Q}, I^{F}, I^{N}, I^{q}, \frac{I^{f},}{\Delta \tau} I^{\eta}, I^{m}$, $I^{\tau}$. Чтение условий $\underline{\Delta m}, \overline{\Delta m}, \underline{\Delta \tau}, \overline{\Delta \tau}$ на скачки [17].

4. Чтение $p \in[1 ;+\infty], \varepsilon>0$ и набора $\left(\delta^{m}\right.$, $\left.\delta^{\tau}, \delta^{i: q}, \delta^{a: q}, \delta^{i: f}, \delta^{a: f}\right)$. Определение по (14), (32) вида задачи на каждом фрагменте. Вычисление величин (15), (16), (33), (34).

5. Диагностика (учитываются условия только порядков $k=0$ и 1) непустоты множества допустимых управлений: построение [3] пробных

$$
\underline{m}, \bar{m}, \underline{\tau}, \bar{\tau} \text {. }
$$

6. Чтение фрагментов функций $m, \tau, q^{\vee}$, $f^{\vee}, \varphi$. Диагностика элементов на соответствие $I^{m}, I^{\tau}, I^{q}, I^{f}$. Диагностика $\varphi$.

7. При $M_{\infty} \geq 10$ чтение $s$, при $M_{\infty}<10$ установка $s=0$.

8. Вычисление $[9,16]$ для заданных фрагментов функций $m, \tau$, дополненных фрагментами (57), и $s$ оценок 
Обратные задачи тепломассообмена на проницаемых поверхностях ГЛА. V. ...

${ }_{-} Q_{-},{ }^{-} Q^{-},{ }_{-} F_{-},{ }^{-} F^{-},{ }_{-} N_{-},{ }^{-} N^{-}$

на $X_{t o t}, X_{\ell}$. Диагностика учитываемых значений $Q, \bar{Q}, \underline{F}, \bar{F}, \underline{N}, \bar{N}$ на соответствие (58).

9. Если $r>1$, то чтение постановки С3: простая или сложная. Для сложной СЭЗ диагностика $\delta_{\ell}^{a}$.

10. При $r=1$ решение задачи в соответствии с [1]. При $r>1$ решение С3 в соответствии с п. 2.3, п. 2.4, п. 3.3, п. 3.4.

5.2. Замечания. 1) Сохранённое решение $m^{\sim}$ и/или $\tau^{\sim}$ может быть на Шаге 6 прочитано в качестве начального приближения для задачи с изменёнными $R, \underline{H}, M_{\infty}, X^{\times}, X_{(r)}^{\wedge}$, $I^{Q}, \ldots, I^{\eta}, I^{m}, I^{\tau}, \underline{\Delta m}, \ldots, \overline{\Delta \tau}, \varepsilon, p, m, \tau, s$, $q^{\vee}, f^{\vee}, \varphi$ и/или типом постановки (простая/ сложная, интерполяционная/аппроксимационная).

2) Сохранённые результаты $m^{\sim}, \tau^{\sim}, q^{\sim}, f^{\sim}$, $\eta^{\sim}$ используются для создания моделей управляющих параметров $m, \tau$, моделей наблюдаемых параметров $q^{\vee}, f^{\vee}, \eta^{\vee}$ и моделей ограничений $I^{m}, I^{\tau}, I^{q}, I^{f}, I^{\eta}$.

3) Сохранённые тотальные и парциальные результаты $Q^{\sim}, F^{\sim}, N^{\sim}$ используются для создания соответствующих моделей ограничений $I^{Q}, I^{F}, I^{N}$.

4) Сохранённые $m^{\sim}, \tau^{\sim}$ и/или $q^{\sim}, f^{\sim}, \eta^{\sim}$ определены для сеток $X^{\wedge}, X^{\vee}$, на которых были получены. Для подготовки их к использованию на иных сетках применяется специальная программа преобразования элементов [17].

\section{ЗАКЛЮЧЕНИЕ}

Введение фрагментации участка управления позволяет явным образом учесть конструкторские и газодинамические ограничения в задачах математического моделирования управления тепломассоообменом и трением в ламинарном пограничном слое на проницаемых цилиндрических и сферических поверхностях гиперзвуковых летательных аппаратов. Проанализированы возможности смешивания разнообразных типов задач управления (прямых и обратных, экстремальных и неэкстремальных, в интерполяционной и аппроксимационной постановках) на фрагментах. Введение фрагментации позволяет решать задачи (AO3, CO3, ГПЭ3, ОЭЗ) в простой постановке и получать близкие к оптимальным управления, которые могут быть использованы в качестве начальных приближений для решения этих задач в сложной постановке. Изучению результатов экспериментов, проведённых согласно вычислительным схемам, предложенным в данной работе, будет посвящено её продолжение.

\section{Работа выполнена:}

а) при государственной поддержке научньхх исследований, проводимьх под руководством ведущих учёных в российских вузах (ведущий учёныци - С. А. Исаев, КНИТУ-КАИ, 2. Казань) по гранту Правительства России № 14.Z50.31.0003;

б) в рамках Государственного задания Министерства образования и науки Российской Федерациии № 9.3236.2017/4.6.

\section{СПИСОК ЛИТЕРАТУРЫ}

1. Бильченко Г. Г., Бильченко Н. Г. Обратные задачи тепломассообмена на проницаемых поверхностях гиперзвуковых летательных аппаратов. IV. Классификация задач на всём участке управления / Г. Г. Бильченко, Н. Г. Бильченко // Вестник Воронеж. гос. унта. Сер. Системный анализ и информационные технологии. - 2018. - № 3. - С. 5-12. 
Бильченко Григорий Григорьевич - канд. физ.-мат. наук, научный сотрудник лаборатории моделирования физико-технических процессов (при кафедре теплотехники и энергетического машиностроения) Казанского национального исследовательского технического университета (КНИТУ-КАИ) им. А. Н. Туполева.

Тел.: +7-905-319-1843

E-mail: <ggbil2@gmail.com>

Бильченко Наталья Григорьевна - канд. физ.-мат. наук, научный сотрудник лаборатории моделирования физико-технических процессов (при кафедре теплотехники и энергетического машиностроения) Казанского национального исследовательского технического университета (КНИТУ-КАИ) им. А. Н. Туполева.

Тел.: +7-905-319-1842

E-mail: <bilchnat@gmail.com>
Bilchenko Grigorij Grigorievich - Candidate of Science in Physics and Mathematics, Researcher of Laboratory of Modeling of Physical and Technical Processes, Department of Heat Engineering and Power Engineering Machinery, Kazan National Research Technical University (KNRTU-KAI) named after A. N. Tupolev.

Tel.: +7-905-319-1843,

E-mail: <ggbil2@gmail.com>

Bilchenko Natalya Grigorievna - Candidate of Science in Physics and Mathematics, Researcher of Laboratory of Modeling of Physical and Technical Processes, Department of Heat Engineering and Power Engineering Machinery, Kazan National Research Technical University (KNRTU-KAI) named after A. N. Tupolev.

Tel.: +7-905-319-1842,

E-mail: <bilchnat@gmail.com> 